\title{
Microbial Degradation Behavior in Seawater of Polyester Blends Containing Poly(3-hydroxybutyrate- co-3-hydroxyhexanoate) (PHBHHx)
}

\author{
Hitoshi Sashiwa ${ }^{1, *}$, Ryuji Fukuda ${ }^{1}$, Tetsuo Okura ${ }^{2}$, Shunsuke Sato ${ }^{3}$ and Atsuyoshi Nakayama ${ }^{4}$ \\ 1 BDP Group, New Buisiness Development Department, Kaneka Co., 5-1-1, Torikai-Nishi, Settsu, \\ Osaka 566-0072, Japan; Ryuji.Fukuda@kaneka.co.jp \\ 2 BDP Processing Technology Development Team, Plastics Molding \& Processing Technology Development \\ Group, Kaneka Co., 5-1-1, Torikai-Nishi, Settsu, Osaka 566-0072, Japan; Tetsuo.Okura@kaneka.co.jp \\ 3 BDP Group, Biotechnology Development Laboratories, Kaneka Co., 8-1 Miyamae-cho, Takasago, \\ Hyogo 676-8688, Japan; Shunsuke.Sato@kaneka.co.jp \\ 4 Biomolecule Design Research Group, Biomedical Research Institute, National Institute of Advanced \\ Industrial Science and Technology (AIST), 1-8-31, Midorigaoka, Ikeda, Osaka 563-8577, Japan; \\ a.nakayama@aist.go.jp \\ * Correspondence: Hitoshi_Sashiwa@kn.kaneka.co.jp; Tel.: +81-72-653-8335
}

Received: 29 November 2017; Accepted: 15 January 2018; Published: 17 January 2018

\begin{abstract}
The microbial degradation behavior of poly(3-hydroxybutyrate-co-3-hydroxyhexanoate) (PHBHHx) and its compound with several polyesters such as poly(butylene adipate-co-telephtharate) (PBAT), poly(butylene succinate) (PBS), and polylactic acid (PLA) in seawater was tested by a biological oxygen demand (BOD) method. PHBHHx showed excellent biodegradation in seawater in this study. In addition, the biodegradation rate of several blends was much influenced by the weight ratio of PHBHHx in their blends and decreased in accordance with the decrement of PHBHHX ratio. The surface morphology of the sheet was important factor for controlling the biodegradation rate of PHBHHx-containing blends in seawater.
\end{abstract}

Keywords: poly(3-hydroxybutyrate-co-3-hydroxyhexanoate) (PHBHHx); blendsurface morphology; microbial; degradation; seawater

\section{Introduction}

In recent years, there is increasing interest in marine pollution owing to the effect of microplastics [1,2]. Microplastics are defined as particles of less than $5 \mathrm{~mm}$ in size and depending on the plastic, it has been shown that various chemicals and toxic blends can be absorbed onto their surface [3]. For this, it is great concern that these blends could be accumulated into zooplankton, fish, and finally to mammals through the food chain. Therefore, the dynamics of production, distribution, diffusion, and degradation of microplastics in marine environments are receiving a lot of attention.

One of the possible solutions toward this problem is focused on the application of biodegradable plastics. Polyhydroxyalkanoates (PHAs) have the most promising biodegradable properties amongst the class of several biodegradable plastics [4,5]. Since PHA plastics degrade and disappear in seawater, toxic blends bound microplastics have no existence in marine environment [6]. Among the PHAs, poly(3-hydroxybutyrate-co-3-hydroxyhexanoate) (PHBHHx, Figure 1) shows excellent flexibility, because the 3-hydroxyhexanoate (3-HHx) unit (Figure 1b) is not taken into the crystallized 3-hydroxybutyrate (3-HB) unit (Figure 1a) in the crystal structure. Therefore, the crystallinity of this polymer can be controlled by changing the percentage of the 3-HHx unit [7]. 


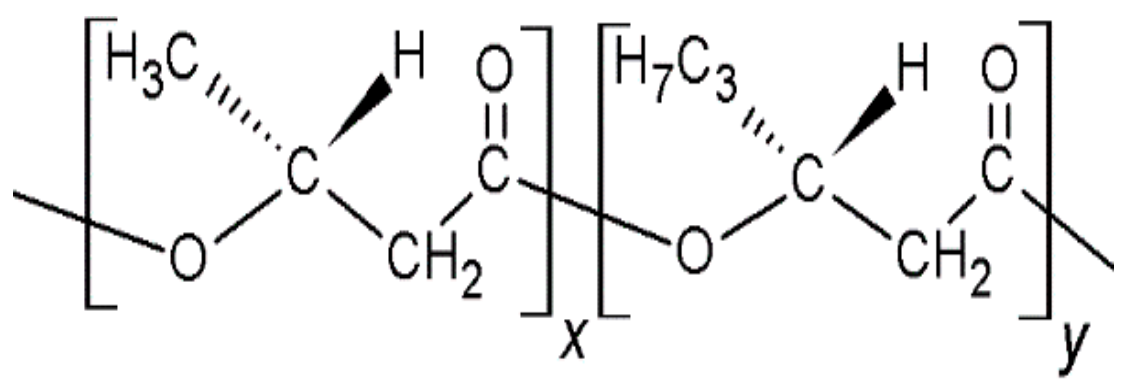

(a)

(b)

Figure 1. Chemical structure of poly(3-hydroxybutyrate-co-3-hydroxyhexanoate) (PHBHHx). (a) hydroxybutyrate (3-HB) unit; (b) 3-hydroxyhexanoate (3-HHx) unit.

PHAs including PHBHHx show good biodegradation properties in soil, as for other biodegradable polyesters such as poly(butylene adipate-co-telephtharate) (PBAT), poly(butylene succinate) (PBS), poly(butylene succinate-co-adipate) (PBSA), polycaprolactone (PCL), etc. [8-10]. Especially, it has been reported that PHAs such as PHB and poly(3-hydroxybutyrate-co-valerate) (PHBV) or PCL showed excellent biodegradation in seawater [11-16]. By contrast, polylactic acid (PLA), which is one of the most utilized biodegradable polyesters, shows poor degradability under ambient conditions, although it is possible to degrade under industrial composting conditions [17]. On the other hand, PHAs are polyesters biologically synthesized by various microorganisms from renewable resources such as saccharides, vegetable oils, glycerol, and others. PHA-degrading bacteria have been isolated from most environments and produce enzymes (extracellular PHA depolymerase) to degrade PHAs under both anaerobic and aerobic conditions. In general, PHAs include PHBHHx showed, blends with other polyesters such as PBAT, PLA, and PBS were carried out to make up the mechanical properties [10]. Therefore, the biodegradability of these blends in seawater needs to be investigated.

In this study, we investigated the effect on seawater biodegradability of different highly biodegradable (as PBAT and PBS) and poorly biodegradable (as PLA) polyesters in the blends (powder and sheets) with PHBHHx at different ratios. Because PHBHHx shows excellent flexibility and heat resistant properties, $\mathrm{PHBHHx}$ is used in not only its independent form but also as blends with several biodegradable polyesters. Moreover, the effect of surface morphology of PHBHHx/PBAT and $\mathrm{PHBHHx} /$ PLA sheets was also investigated in this study.

\section{Results and Discussion}

\subsection{Biodegradation of $\mathrm{PHBHH} x$ and $\mathrm{PHBHH} x / \mathrm{PBAT}$ Blends}

In this study, $11 \mathrm{~mol} \%$ of $3-\mathrm{HHx}$ unit content of PHBHHx was used. Table 1 shows the bending elastic modulus and the molecular weight of polymers used in this study before and after biodegradation in seawater for 28 days. PBAT, PBS, and PLA, which were not shown microbial metabolism estimated by biological oxygen demand (BOD) test, showed slight decrease of molecular weight by hydrolysis in seawater. On the other hand, $\mathrm{PHBHHx}$, which showed microbial metabolism, did not show molecular weight change. This phenomenon could be explained as follows: first the surface of PHBHHx was hydrolyzed by microbial enzymes, and then seawater soluble oligomers of PHBHHx were metabolized by microorganisms in seawater. Since it has been shown that PHBHHx showed high resistance of hydrolysis in comparison with those of PBAT, PBS, and PLA, the remained PBHHx would still keep the high molecular weight. 
Table 1. The molecular weight of polymers (sheet shape) used in this study before and after biodegradation in seawater for 28 days.

\begin{tabular}{cccccccc}
\hline \multirow{2}{*}{ Polymer } & \multicolumn{1}{c}{ Bending Elastic Modulus } & \multicolumn{3}{c}{ Before Biodegradation } & \multicolumn{2}{c}{ After 28 Days Biodegradation } \\
\cline { 2 - 8 } & $\mathbf{M P a}$ & $\mathbf{M}_{\mathbf{n}}$ & $\mathbf{M w}$ & $\mathbf{M w} / \mathbf{M}_{\mathbf{n}}$ & $\mathbf{M}_{\mathbf{n}}$ & $\mathbf{M w}$ & $\mathbf{M w} / \mathbf{M}_{\mathbf{n}}$ \\
\hline PHBHHx & 850 & 280,000 & 550,000 & 2.0 & 280,000 & 530,000 & 1.9 \\
PBAT & 110 & 63,000 & 120,000 & 1.9 & 55,000 & 110,000 & 2.0 \\
PBS & 650 & 81,000 & 150,000 & 1.9 & 77,000 & 126,000 & 1.6 \\
PLA & 3190 & 100,000 & 160,000 & 1.6 & 94,000 & 140,000 & 1.5 \\
\hline
\end{tabular}

Figure 2 shows the typical time courses of the biodegradation of PHBHHx and PHBHHx/PBAT blends (powder form) in seawater as indicated by their biological oxygen demand (BOD). The biodegradation of PHBHHx gradually increased with the degradation time. The pure PHBHHx showed excellent biodegradability in seawater in comparison with the original PBAT, whose biodegradability was quite low level. The degree of biodegradation therefore showed much dependence on the effect of the weight ratio in the blend. The biodegradation was decreased with the ratio of $\mathrm{PHBHHx}$.

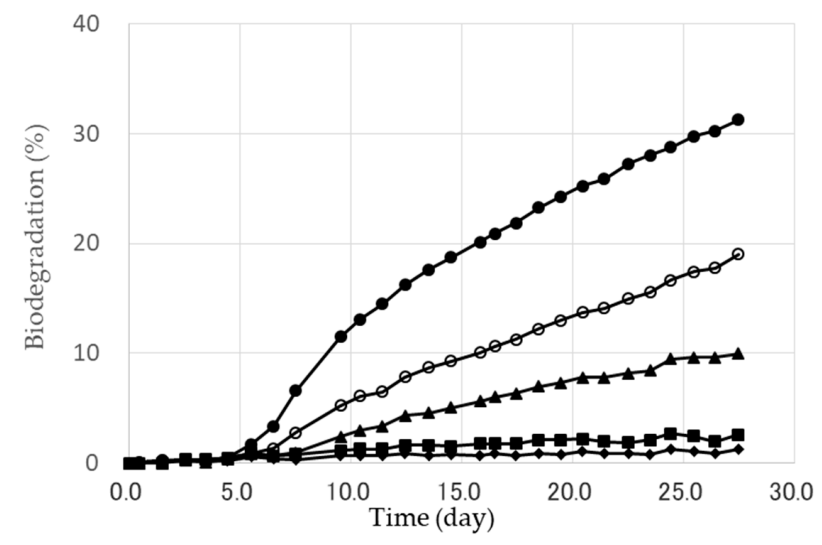

Figure 2. The biodegradation of PHBHHx and PHBHHx/PBAT blends (powder shape) in seawater.

๑: PHBHHx, $\bigcirc:$ PHBHHx/PBAT $=80 / 20$ (wt/wt), $\Delta:$ PHBHHx $/$ PBAT $=60 / 40$ (wt $/ w t)$,

口: $\mathrm{PHBHHx} / \mathrm{PBAT}=40 / 60(\mathrm{wt} / \mathrm{wt})$, : PBAT.

The biodegradation degree (\%) in seawater after 28 days is summarized in Table 2. The particle size distribution of all powder form of samples were around 24-2000 $\mu \mathrm{m}$.

To confirm only the PHBHHx component degrade in the blends, the content (wt \%) of PHBHHx in the blends were analyzed by ${ }^{1} \mathrm{H}-\mathrm{NMR}$ analysis before and after biodegradation (Figure S1). The contents of PHBHHx before biodegradation were a little bit changed after the melt exclusion process. As shown in Table 2, the contents of PHBHHx were slightly decreased after biodegradation, which means that PHBHHx component surely degraded with seawater.

Table 2. The biodegradation degree (\%) of PHBHHx/PBAT blends in seawater after 28 days.

\begin{tabular}{|c|c|c|c|c|c|c|}
\hline \multirow[t]{2}{*}{ Entry } & \multirow{2}{*}{$\begin{array}{c}\text { РНВНHх/РBAT } \\
\text { Added (wt/wt) }\end{array}$} & \multirow[t]{2}{*}{ Form } & \multirow{2}{*}{$\begin{array}{l}\text { Average Particle } \\
\text { Size }(\mu \mathrm{m})\end{array}$} & \multirow[t]{2}{*}{ Biodegradation (\%) } & \multicolumn{2}{|c|}{$\begin{array}{l}\text { Content of PHВНHx } \\
\text { in Blends (wt \%) }\end{array}$} \\
\hline & & & & & Before & After \\
\hline 1 & $100 / 0$ & Powder & 440 & 31 & - (a) & - \\
\hline 2 & $80 / 20$ & Powder & 380 & 19 & 83 & 80 \\
\hline 3 & $60 / 40$ & Powder & 400 & 10 & 66 & 61 \\
\hline 4 & $40 / 60$ & Powder & 400 & 3 & 47 & 46 \\
\hline 5 & $0 / 100$ & Powder & 470 & 1 & - & - \\
\hline
\end{tabular}

(a) Not tested. 


\subsection{Biodegradation of $\mathrm{PHBHH} / \mathrm{PBS}$ Blends}

The biodegradation degree (\%) of PHBHHx/PBS blends in seawater after 28 days is summarized in Table 3. In the case of PHBHHx/PBS, the biodegradability also showed much dependence on the PBS ratio in the blends. On comparing with the relative rates (calcd. and found), $\mathrm{PHBHHx} / \mathrm{PBS}=$ $80 / 20$ (entry 2) was not found to inhibit the biodegradation rate. However, the $60 / 40$ and $40 / 60$ blends (entries 3 and 4) showed the same level of inhibition as the PBAT blends. These results suggest that a high amount of PBS also inhibits the biodegradability of PHBHHx. In these cases, PHBHHx components were surely decreased after biodegradation. The content (wt \%) of PHBHHx in the blends were analyzed by ${ }^{1} \mathrm{H}-\mathrm{NMR}$ analysis before and after biodegradation (Figure S3). And Figure S4 shows the time courses of the biodegradation of PHBHHx/PLA blends (powder form) in seawater.

Table 3. The effect of mixture ratio of PHBHHx/PBS blends on the biodegradation in seawater after 28 days.

\begin{tabular}{ccccccc}
\hline Entry & $\begin{array}{c}\text { PHBHHx/PBS } \\
(\mathbf{w t} / \mathbf{w t})\end{array}$ & Form & $\begin{array}{c}\text { Average Particle } \\
\text { Size }(\boldsymbol{\mu m})\end{array}$ & Biodegradation (\%) & \multicolumn{2}{c}{$\begin{array}{c}\text { Content of PHBHHx } \\
\text { in Blends (wt \%) }\end{array}$} \\
\cline { 6 - 7 } & & & & 51 & Before & After \\
\hline 1 & $100 / 0$ & Powder & 440 & 41 & - (a) & - \\
2 & $80 / 20$ & Powder & 370 & 18 & 61 & 57 \\
3 & $60 / 40$ & Powder & 420 & 5 & 42 & 38 \\
4 & $40 / 60$ & Powder & 390 & 1 & - & - \\
5 & $0 / 100$ & Powder & 310 & (a) Not tested.
\end{tabular}

\subsection{Biodegradation of PHBHHx/PLA Blends}

Figure 3 shows the time courses of the biodegradation of PHBHHx/PLA blends (powder form) in seawater. The original PLA showed quite low biodegradability in seawater, as for the PBAT and PBS. However the biodegradation of PHBHHx/PLA blends showed less dependence on the PLA ratio. The biodegradation degree (\%) in seawater after 28 days is summarized in Table 4 . In these cases, PHBHHx components were surely decreased after biodegradation. The reason of relatively high biodegradation the PHBHHx/PLA blends of 80/20, 60/40, and 40/60 is discussed later (Section 2.5). The content (wt \%) of PHBHHx in the blends were analyzed by ${ }^{1} \mathrm{H}-\mathrm{NMR}$ analysis before and after biodegradation (Figures S2-1 and S2-2). And Figure S5 shows the particle size distribution of PHBHHx/PLA blend powders.

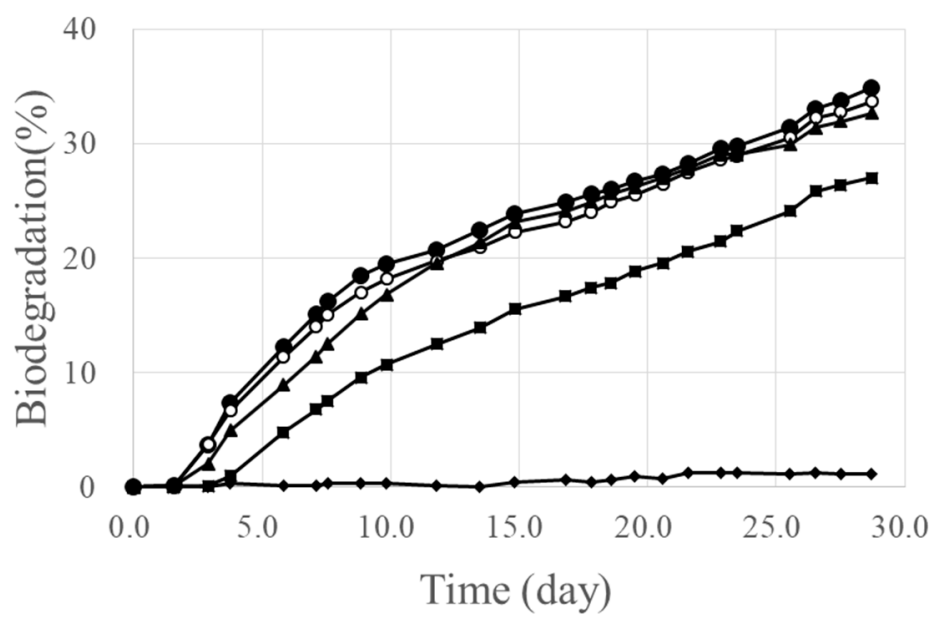

Figure 3. The biodegradation of PHBHHx and PHBHHx/PLA blends (powder shape) in seawater. ๑: PHBHHx, $\bigcirc:$ PHBHHx/PLA = 80/20 (wt/wt), $\mathbf{\Delta}:$ PHBHHx/PLA = 60/40 (wt/wt), ם: PHBHHx/PLA $=40 / 60$ (wt $/ w t), \diamond:$ PLA. 
Table 4. The effect of mixture ratio of PHBHHx/PLA blends on the biodegradation in seawater after 28 days.

\begin{tabular}{|c|c|c|c|c|c|c|}
\hline \multirow{2}{*}{ Entry } & \multirow{2}{*}{$\begin{array}{l}\text { PHBHHx/PLA } \\
\text { (wt/wt) }\end{array}$} & \multirow[t]{2}{*}{ Form } & \multirow{2}{*}{$\begin{array}{l}\text { Average Particle } \\
\text { Size }(\mu \mathrm{m})\end{array}$} & \multirow[t]{2}{*}{ Biodegradation (\%) } & \multicolumn{2}{|c|}{$\begin{array}{l}\text { Content of РНВННx } \\
\text { in Blends (wt \%) }\end{array}$} \\
\hline & & & & & Before & After \\
\hline 1 & $100 / 0$ & Powder & 440 & 34 & - (a) & - \\
\hline 2 & $80 / 20$ & Powder & 420 & 33 & 82 & 77 \\
\hline 3 & $60 / 40$ & Powder & 430 & 32 & 64 & 62 \\
\hline 4 & $40 / 60$ & Powder & 440 & 26 & 51 & 45 \\
\hline 5 & $0 / 100$ & Powder & 490 & 1 & - & - \\
\hline
\end{tabular}

\subsection{Effect of the Morphology of PHBHHx/PBAT Compound on the Biodegradation}

Table 5 shows the effect of the form of the PHBHHx/PBAT blends on the biodegradation rate in seawater after 28 days. In the most cases, the sheet forms (entries 2 and 4 ) showed less biodegradability in comparison with those of powder form. To clarify this reason for these difference, transmission electron microscope (TEM) and scanning electron microscope (SEM) analyses were performed. Figure 4 shows the SEM image of the RuO4 stained PHBHHx/PBAT $=80 / 20$ and $60 / 40$ blend sheets surface before biodegradation. The PBAT was the brighter part owing to a higher scattering intensity of the electron beam by the attachment of $\mathrm{RuO}_{4}$ to the phenyl group in PBAT compared with the PHBHHx component. Despite the high PHBHHx fraction in PHBHHx/PBAT $=80 / 20$ and $60 / 40$, a large proportion of the PBAT component was observed at the surface of these sheets, which would be caused by the lower molecular weight of PBAT layer tends to exposure at the surface area under preparing T-die sheet. This would explain the decrease of biodegradation for the PBAT blends because the PBAT at the surface restricted microorganial access to PHBHHx.

Figure 5 shows the TEM photograph of a cross section of the PHBHHx/PBAT $=80 / 20$ and $60 / 40$ sheets before biodegradation. The PBAT component was darks owing to less permeability of electron beam through the attachment of $\mathrm{RuO}_{4}$ to the phenyl group in the PBAT compared with the PHBHHx part. The PBAT layer structure (black parts) was arranged like an annual ring of tree for the $60 / 40$ blend in comparison with that of $80 / 20$ which was more irregular. This difference means that the biodegradation was more inhibited for the $60 / 40$ blend. Since the PBAT did not show biodegradation, the biodegradation of PHBHHx/PBAT blends was highly inhibited at the PBAT layer. Overall, these morphological effects cause the decrease of biodegradability in PHBHHx/PBAT sheet.

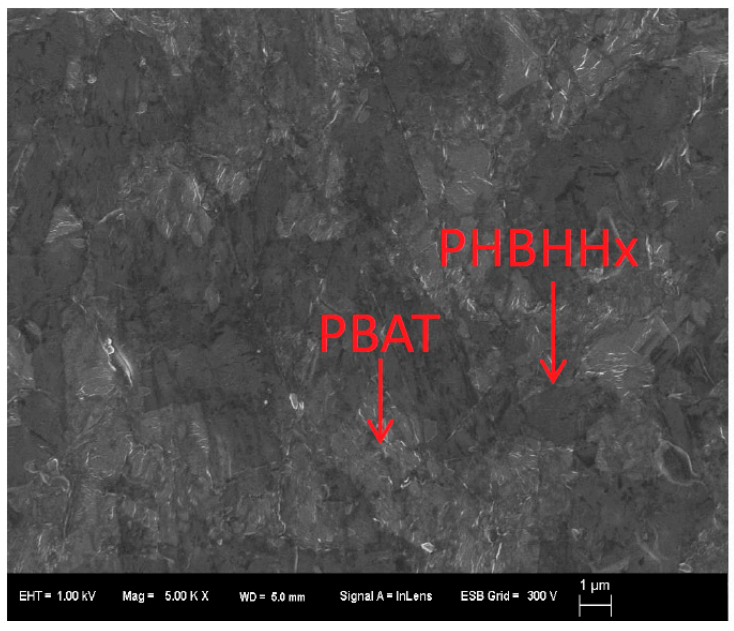

(a)

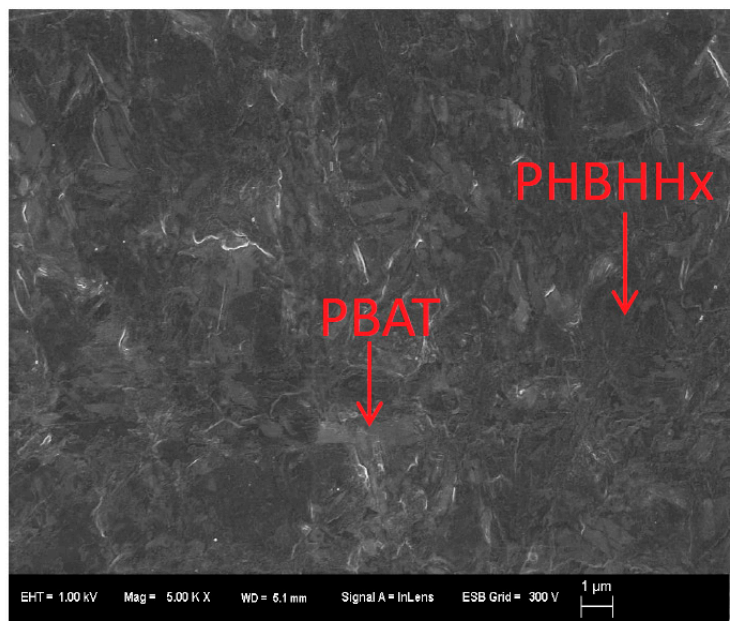

(b)

Figure 4. Typical scanning electron microscope (SEM) micrographs of the blend of $\mathrm{PHBHHx} / \mathrm{PBAT}=$ $80 / 20$ (a) and $60 / 40$ (b) sheet surface $(\times 5000)$ before biodegradation. The scale bar length is $1 \mu \mathrm{m}$. 


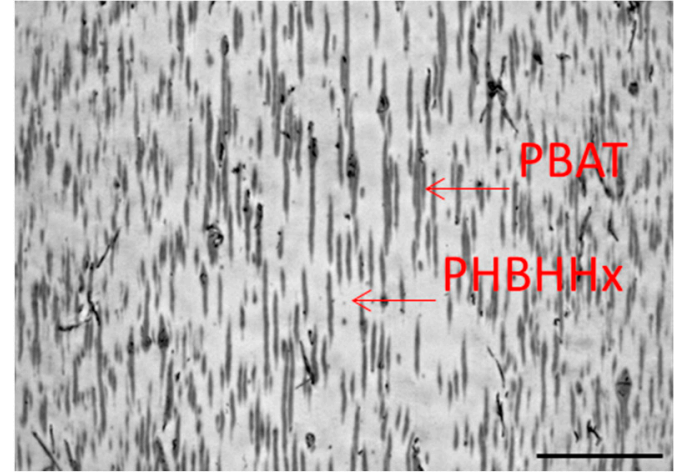

(a)

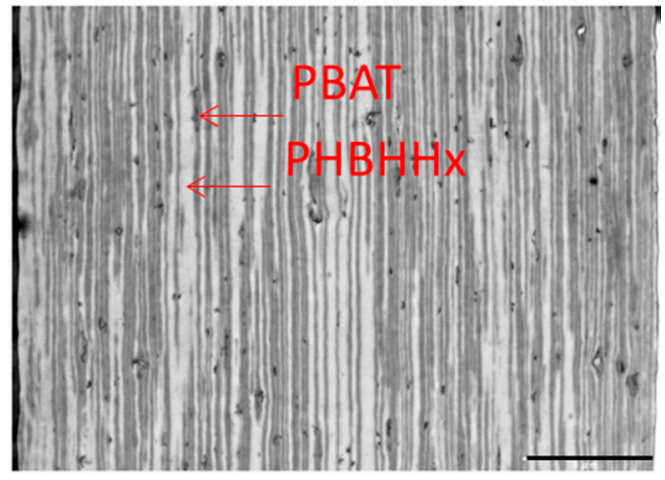

(b)

Figure 5. Typical transmission electron microscope (TEM) micrographs of the cross section of a sheet of PHBHHx/PBAT = 80/20 (a) and 60/40 (b) before biodegradation. The scale bar length is $5 \mu \mathrm{m}$.

Table 5. The effect of morphology of PHBHHx/PBAT blends on the biodegradation in seawater after 28 days.

\begin{tabular}{|c|c|c|c|c|c|}
\hline Entry & Mixture & Ratio & Form ${ }^{(a)}$ & Biodegradation (\%) & Effect of Morphology \\
\hline 1 & PHBHHx/PBAT & $80 / 20$ & Powder & 19 & \\
\hline 2 & & $80 / 20$ & Sheet & 10 & $\mathrm{O}^{(\mathrm{b})}$ \\
\hline 3 & & $60 / 40$ & Powder & 10 & \\
\hline 4 & & $60 / 40$ & Sheet & 2 & 0 \\
\hline 5 & & $40 / 60$ & Powder & 3 & \\
\hline 6 & & $40 / 60$ & Sheet & 1 & Non degradable \\
\hline
\end{tabular}

(a) The thickness of each sheets is $20 \mu \mathrm{m}$; ${ }^{(b)} \bigcirc$, effect of morphology was observed.

\subsection{Effect of the Morphology of PHBHHx/PLA Compound on the Biodegradation}

Table 6 shows the effect of the form of the PHBHHx/PLA blends on their biodegradation in seawater after 28 days. In these cases, the sheet forms (entries 4 and 6) also showed less biodegradability in comparison with those in powder forms. In the previous paper, the PLA/PHA biodegradable blends for pneumothermic fablication of nonwoven was reported [18]. However, there is no information on the morphology of PHA and PLA blends. To clarify the reason for this, scanning electron microscope (SEM) analysis were performed.

Table 6. The effect of morphology of PHBHHx/PLA blends on the biodegradation in seawater after 28 days.

\begin{tabular}{cccccc}
\hline Entry & Mixture & Ratio & Form $^{(\mathbf{a})}$ & Biodegradation (\%) $^{\text {Effect of Morphology }}$ \\
\hline 1 & PHBHHx/PLA & $80 / 20$ & Powder & 33 & \\
2 & & $80 / 20$ & Sheet & 42 & None \\
3 & & $60 / 40$ & Powder & 32 & None \\
4 & & $60 / 40$ & Sheet & 20 & \\
5 & $40 / 60$ & Powder & 26 & $\bigcirc^{(b)}$ \\
6 & & $40 / 60$ & Sheet & 8 & \\
\hline
\end{tabular}

(a) The thickness of each sheets is $20 \mu \mathrm{m}$; ${ }^{(b)} \bigcirc$, effect of morphology was observed.

Figure 6 shows the typical SEM photograph of the sheet surface of the PHBHHx/PLA 80/20 blend before and after biodegradation in seawater. A lot of hollows were observed on the surface of this highly biodegradable sheet after biodegradation (Figure 6b). By contrast, the less biodegradable sheet $(60 / 40)$ had a relatively flat structure after seawater exposure (Figure 6d). This suggests that the surface of the less degradable sheet $(60 / 40)$ was more covered with the low-degradable PLA component than PHBHHx component. Therefore, the masking effect of these blend sheets could be the reason for less 
biodegradability. In conclusion, the surface morphology of the melt blend sheets was an important factor for controlling the biodegradation in seawater.

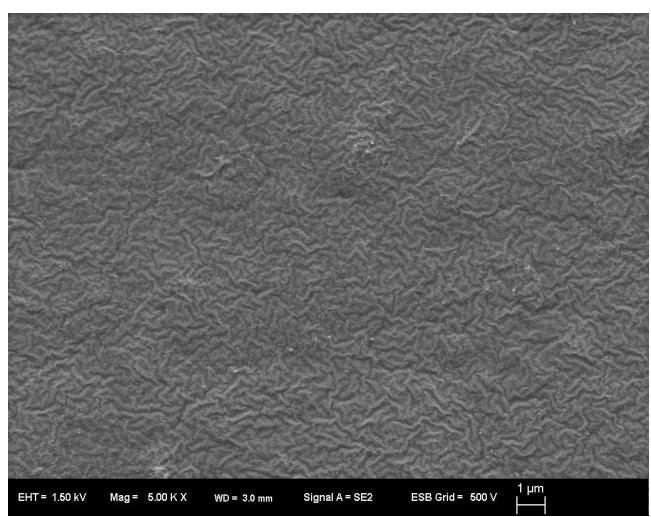

(a)

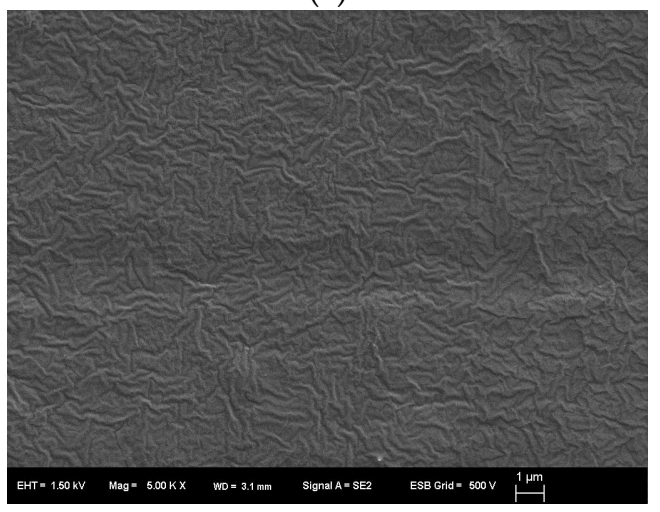

(c)

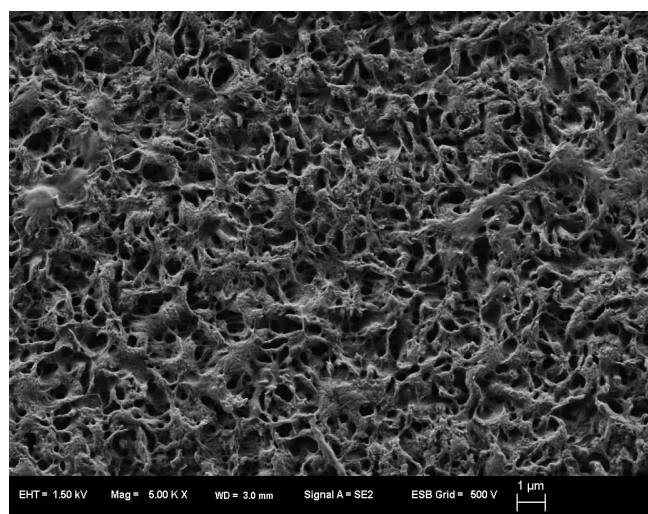

(b)

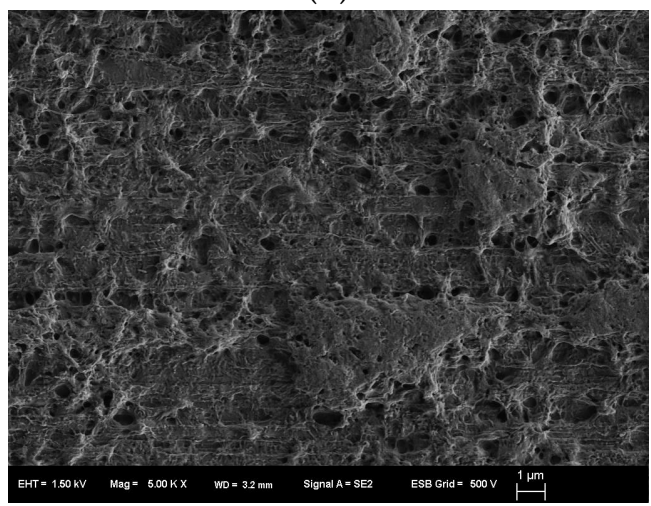

(d)

Figure 6. SEM photograph $(\times 5000)$ of the blend sheets of $($ a) $\mathrm{PHBHHx} / \mathrm{PLA}=80 / 20$ before, (b) $\mathrm{PHBHHx} / \mathrm{PLA}=80 / 20$ after, (c) $\mathrm{PHBHHx} / \mathrm{PLA}=60 / 40$ before, (d) PHBHHx/PLA = 60/40 after biodegradation in seawater. The scale bar length is $1 \mu \mathrm{m}$.

Figure 7 shows the surface the fourier-transform infrared spectroscopy (FT-IR) by attenuated total reflectance (ATR) spectroscopy of sheets of PHBHHx, PLA, and their blends before and after 28 days of biodegradation in seawater. The peak areas of the $\mathrm{C}=\mathrm{O}$ stretching bands at $1756 \mathrm{~cm}^{-1}$ corresponding to the PLA component and those at $1721 \mathrm{~cm}^{-1}$ corresponding to the PHBHHx component were compared before and after biodegradation. As shown in Figure 7, the peak area corresponding to the PHBHHx decreased and the PLA area increased after biodegradation in seawater. In the case of PHBHHx/PLA = 60/40 and 40/60, especially, a significant change was observed in Figure 7c-f. This suggests that the ratio of PLA components increased at the surface of the blend sheets after biodegradation, indicating that the PHBHHx components degrade preferentially on the surface of PHBHHx/PLA blend sheets. As shown in Table 1, PLA shows higher bending elastic modulus than that of PHBHHx. Therefore, the reason of high biodegradation the PHBHHx/PLA blends powders would be caused by the increment of surface of $\mathrm{PHBHHx}$, which is formed some clacking at the freeze pulverization process. These increment of the surface area of PHBHHx would be supported by the clear decrease of surface PHBHHx component by FT-IR spectroscopic analyses. 


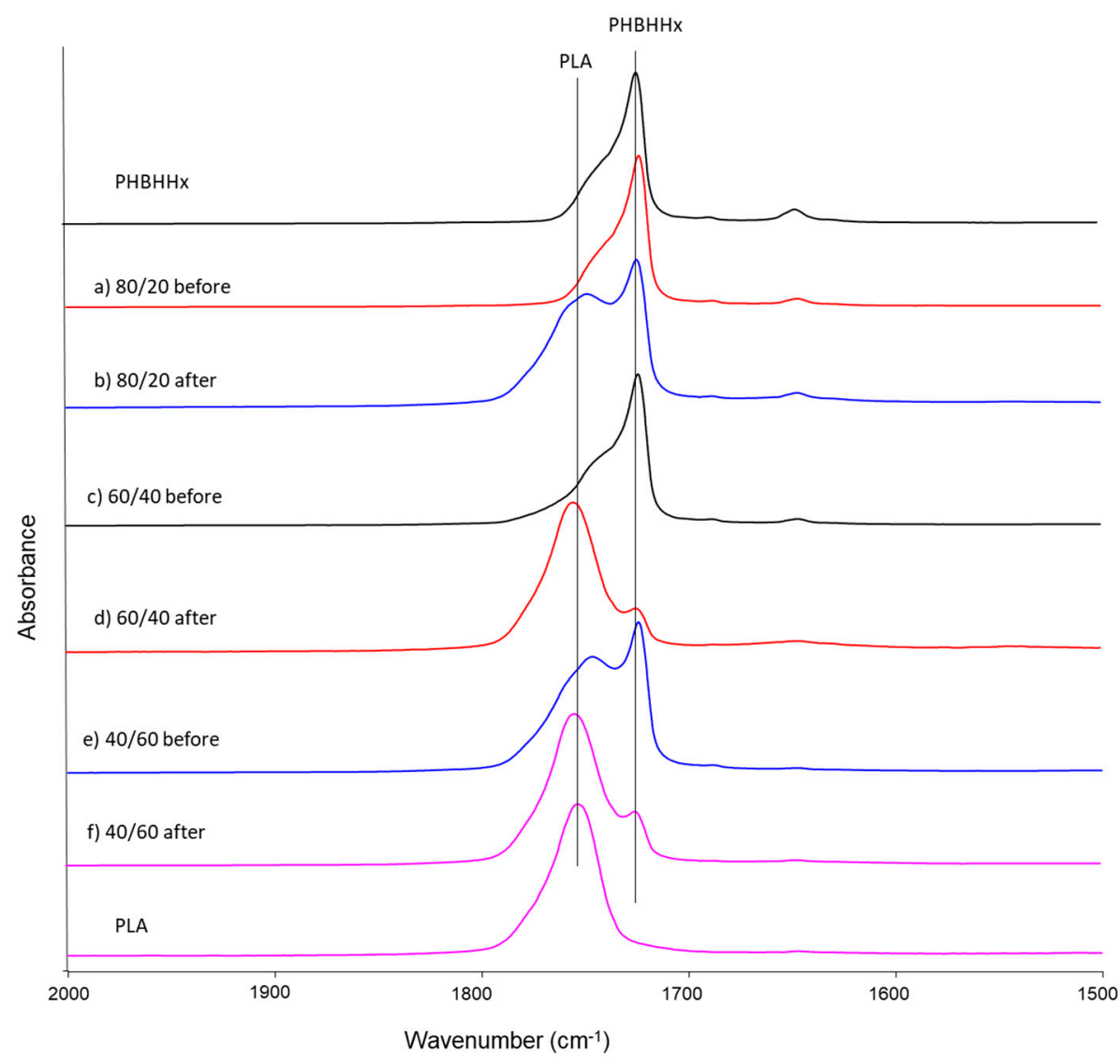

Figure 7. Fourier-transform infrared spectroscopy (FT-IR) by attenuated total reflectance (ATR) spectra of sheets of PHBHHx, PLA, and their blends before and after biodegradation in seawater.

\section{Materials and Methods}

\subsection{Materials}

A commercial grade of PHBHHx (3-HHx unit content $=11 \mathrm{~mol} \%$, X151A, OKANEKA Biodegradablepolymer ${ }^{\mathrm{TM}} \mathrm{PHBH}$, Kaneka Co., Osaka, Japan) was used in this study. PBAT (Ecoflex C-1200, BASF), PLA (Ingeo 10361D, Nature Works, Minnetonka, MN, USA), and PBS (Bionolle 1020MD, Showa Denko, Tokyo, Japan) were used as obtained. Each blend (PHBHHx/PBAT, PHBHHx/PLA, PHBHHx/PBS) was prepared by a melt extrusion process using TEM26SS (Toshiba Machine, Numazu, Japan) at $140{ }^{\circ} \mathrm{C}$ with $80 \mathrm{rpm}$ for $2 \mathrm{~min}$ to obtain each blended pellets. Each blended pellets were pulverized by freeze pulverization using a SPEX SamplePre 6750 Freezer/Mill (SPEX) to obtain blend powders. The average particle size of each blend powder was measured using a Microtruc MT3300EXII (Nikkiso, Tokyo, Japan). Sheets of the blends at $20 \mu \mathrm{m}$ of thickness were also prepared by a T-die cast extrusion process at $140{ }^{\circ} \mathrm{C}$ for 8 min using a Labo-Plastmill Model $30 \mathrm{C} 150$ (Toyoseiki, Nagano, Japan). The data of each bending elastic modulus was cited from the previous study [10].

\subsection{Biodegradation of Polyesters}

Biodegradation of polyesters with seawater was evaluated using a BOD tester $200 \mathrm{~F}$ (TAITEC Co., Tokyo, Japan) according to the previous report [19]. Seawater was collected at the Osaka-Nanko bay area. The typical procedure is as follows: Seawater $(200 \mathrm{~mL})$, and sample $(30 \mathrm{mg})$ were placed in a fermenter at $27^{\circ} \mathrm{C}$. $\mathrm{Ca}(\mathrm{OH})_{2}$ powder $(1.0 \mathrm{~g})$ was used as a trap for produced $\mathrm{CO}_{2}$ gas. At the prescribed time, the amount of consumed $\mathrm{O}_{2}$ gas was measured. The fermenter, which did not include the sample, was used as a control. The net amount of consumed $\mathrm{O}_{2}$ gas $(\mathrm{mL})$ was evaluated as the 
difference of the sample and control. The theoretical amount of consumed $\mathrm{O}_{2}(\mathrm{~mL})$ means the total $\mathrm{O}_{2}$ that should be consumed on complete degradation of sample. Biodegradation (\%) was calculated as follows:

In the case of $\mathrm{PHBHHx}\left(11 \mathrm{~mol} \%\right.$ of $3-\mathrm{HHx}$ content), the consumption of $\mathrm{O}_{2}$ is the following Equation (1), and the biodegradation is estimated by the Equation (2):

$$
\mathrm{C}_{4.22} \mathrm{H}_{6.44} \mathrm{O}_{2}+4.83 \mathrm{O}_{2} \rightarrow 4.22 \mathrm{CO}_{2}+3.22 \mathrm{H}_{2} \mathrm{O} \text {, }
$$

Biodegradation $(\%)=\left[\right.$ experimentally consumed $\mathrm{O}_{2}(\mathrm{~mL}) /$ theoretical $\left.\mathrm{O}_{2}(\mathrm{~mL})\right] \times 100$.

The consumption of $\mathrm{O}_{2}(\mathrm{~mL})$ without samples was deleted from the experimentally consumed $\mathrm{O}_{2}$ $(\mathrm{mL})$. The biodegradation value $(\%)$ was average value of duplicate data $(N=2)$. All biodegradation data using a BOD tester occurred by the microbial metabolism. Therefore, deionized water without microorganisms does not occur with the $\mathrm{O}_{2}$ consumption.

\subsection{TEM and SEM Analysis}

Transmission electron microscope (TEM) observation of each samples were carried out after treatment with $\mathrm{RuO} 4$ using a Hitachi H-7650 transmission electron microscope (Hitachi High-Technologies, Tokyo, Japan). Scanning electron microscope (SEM) observation of each samples were also carried out after treatment with $\mathrm{RuO} 4$ using Zeiss ULTRAplus scanning electron microscope (Zeiss, Oberkochen, Germany).

\subsection{FT-IR Analysis}

FT-IR spectroscopy of the sample surface by attenuated total reflectance (ATR) spectra was measured using a Perkin Elmer Co., Ltd. (Waltham, MA, USA) model Frontier FT-IR spectrometer.

\section{5. ${ }^{1} \mathrm{H}-\mathrm{NMR}$ Analysis}

${ }^{1} \mathrm{H}-\mathrm{NMR}$ analysis of each blends before and after biodegradation was measured using Brucker $400 \mathrm{MHx}$ NMR spectrometer (Bruker Biospin Co., Yokohama, Japan) in $\mathrm{CDCl}_{3}$ as solvent. The content of PHBHHx, PBAT, and PHB was estimated from the following peak areas; PHBHHx $=5.27 \mathrm{ppm}$ $(\mathrm{CH}), \mathrm{PLA}=5.17 \mathrm{ppm}(\mathrm{CH}), \mathrm{PBAT}=8.12 \mathrm{ppm}\left(\mathrm{C}_{6} \mathrm{H}_{4}\right.$ of aromatic protons $), \mathrm{PBS}=2.64 \mathrm{ppm}\left(\mathrm{CH}_{2}\right.$ of succinate).

\subsection{Molecular Weight Analysis}

The molecular weight (Mn, Mw) of PHBHHx, PBAT, PLA, and PBS was determined by GPC (apparatus, Shimadzu LC-10A, Shimadzu Co., Kyoto, Japan, column, Shodex GPCK-806M, Showa Denko, Tokyo, Japan, eluent, chloroform, injection, $10 \mu \mathrm{L}$, column temperature, $\left.40{ }^{\circ} \mathrm{C}\right) \mathrm{using}$ polystyrene as standard at the concentration of $1.5 \mathrm{mg} / \mathrm{mL}$.

\section{Conclusions}

The PHBHHx sheets and powders showed excellent biodegradability in seawater. The biodegradation of the blends of PHBHHx/PBAT, PHBHHx/PLA, and PHBHHx/PBS was significantly influenced by the weight ratio of their melt blends and decreased in accordance with the decrement of PHBHHx ratio from the BOD tester. The surface morphology of the PHBHHx/PBAT and PHBHHx/PLA blends sheet was an important factor for controlling the biodegradation in seawater based on the SEM and TEM analysis. The PHBHHx component at the surface decreased on degradation in seawater based on the FT-IR (ATR) analysis. From these results, it is clear that the PHBHHx component degraded across a range of blend compositions in seawater.

In this study, PHBHHx was shown to have excellent biodegradability in seawater, but the biodegradation rate of other biodegradable polymers was shown to be much lower than that of 
the PHBHHx. From the results of this study, PHBHHx-containing blends degrade much faster than conventional plastics like PBAT, PBS, and PLA and so on, indicating that PHA blends including $\mathrm{PHBHHx}$ are useful materials for the marine environment.

Supplementary Materials: The following are available online at www.mdpi.com/1660-3397/16/1/34/s1, Figure S1: ${ }^{1} \mathrm{H}-\mathrm{NMR}$ spectra of PHBHHx/PBAT $=80 / 20$, Figure S2-1: ${ }^{1} \mathrm{H}-\mathrm{NMR}$ spectra of PHBHHx $/ \mathrm{PLA}=$ 80/20, Figure S2-2: Enlarged view of the ${ }^{1} \mathrm{H}-\mathrm{NMR}$ spectra of PHBHHx/PLA $=80 / 20$, Figure S3: ${ }^{1} \mathrm{H}-\mathrm{NMR}$ spectra of PHBHHx/PBS = 80/20, Figure S4: The biodegradation of PHBHHx and PHBHHx/PBS blends (powder shape) in seawater, Figure S5: Particle size distribution of PHBHHx/PLA blend powders.

Author Contributions: Hitoshi Sashiwa: Contribution of whole experimental data and whole manuscript preparation, Ryuji Fukuda: Contribution of experimental idea mainly TEM, SEM, and FT-IR analysis and checking manuscript, Tetsuo Okura: Contribution of experimental planning mainly PHBHHx and PBAT, PLA, PBS blends, Shunsuke Sato: Contribution of introduction section mainly biological aspects, Atsuyoshi Nakayama: Experimental contribution of biodegradation using BOD tester.

Conflicts of Interest: The authors declare no conflict of interest.

\section{References}

1. Jambeck, J.R.; Geyer, R.; Wilcox, C.; Siegler, T.R.; Perryman, M.; Andrady, A.; Narayan, R.; Law, K.L. Plastic waste inputs from land into the ocean. Science 2015, 347, 768-771. [CrossRef] [PubMed]

2. Lönnstedt, O.M.; Eklöv, P. Environmentally relevant concentrations of microplastic particles influence larval fish ecology. Science 2016, 352, 1213-1216. [CrossRef] [PubMed]

3. Yeo, B.G.; Takada, H.; Taylor, H.; Ito, M.; Hosoda, J.; Allinson, M.; Connell, S.; Greaves, L.; McGrath, J. POPs monitoring in Australia and New Zealand using plastic resin pellets, and International Pellet Watch as a tool for education and raising public awareness on plastic debris and POPs. Mar. Pollut. Bull. 2015, 101, 137-145. [CrossRef] [PubMed]

4. Doi, Y.; Kitamura, S.; Abe, H. Microbial synthesis and characterization of poly(3-hydroxybutyrate-co3-hydroxyhexanoate). Macromolecules 1995, 28, 4822-4828. [CrossRef]

5. Feng, L.; Watanabe, T.; Wang, Y.; Kichise, T.; Fukuchi, T.; Chen, G.-Q.; Doi, Y.; Inoue, Y. Studies on comonomer compositional distribution of bacterial poly(3-hydroxybutyrate-co-3-hydroxyhexanoate)s and thermal characteristics of their factions. Biomacromolecules 2002, 3, 1071-1077. [CrossRef] [PubMed]

6. Mabrouk, M.M.; Sabry, S.A. Degradation of poly(3-hydroxybutyrate) and its copolymer poly(3hydroxybutyrate-co-3-hydroxyvalerate) by a marine Streptomyces sp. SNG9. Microbiol. Res. 2001, 156, 323-335. [CrossRef] [PubMed]

7. Sato, H.; Murakami, R.; Padermshoke, A.; Hirose, F.; Senda, K.; Noda, I.; Ozaki, Y. Infrared spectroscopy studies of $\mathrm{CH} \cdots \mathrm{O}$ hydrogen bondings and thermal behavior of biodegradable poly(hydroxyalkanoate). Macromolecules 2004, 37, 7203-7213. [CrossRef]

8. Kasuya, K.; Takagi, K.; Ishiwatari, S.; Yoshida, Y.; Doi, Y. Biodegradabilities of various aliphatic polyesters in natural waters. Polym. Degrad. Stab. 1998, 59, 327-332. [CrossRef]

9. Okura, T. Biodegradability of bacterial polymer Aonilex@ in seawater. BioPla J. 2016, 62, 5-9.

10. Sugaya, T. Exploition and development of bacterial polymer Aonilex@. Convertech 2016, 517, 128-131.

11. Yamamoto, M.; Witt, U.; Skuprin, G.; Beimborn, D.; Muller, R.-J. Biodegradable Aliphatic-Aromatic Polyesters: "Ecoflex ${ }^{\circledR "}$ Biopolymers; Doi, Y., Steinbuchel, A., Eds.; Polyesters III Applications and Commercial Products; Wiley-VCH: Weinheim, Germany, 2002; Volume 4, pp. 299-312.

12. Emadian, S.M.; Onay, T.T.; Demirel, D. Biodegradation of bioplastics in natural environments. Waste Manag. 2017, 59, 526-536. [CrossRef] [PubMed]

13. Tachibana, K.; Urano, Y.; Numata, K. Biodegradability of nylon 4 film in a marine environment. Polym. Degrad. Stab. 2013, 98, 1347-1351. [CrossRef]

14. Tallen, C.; Coyne, M.; Froio, D.; Auerbach, M.; Wirsen, C.; Ratto, J.A. A Processing, characterization and marine biodegradation study of melt-extruded polyhydroxyalkanoate (PHA) films. J. Polym. Environ. 2008, 16, 1-11. [CrossRef] 
15. Volova, T.G.; Boyandin, A.N.; Vasiliev, A.D.; Karpov, V.A.; Prudnikova, S.V.; Mishukova, O.V.; Boyarskikh, U.A.; Filipenko, M.L.; Rudnev, V.P.; Xuân, B.B.; et al. Biodegradation of polyhydroxyalkanoates (PHAs) in tropical coastal waters and identification of PHA-degrading bacteria. Polym. Degrad. Stab. 2010, 95, 2350-2359. [CrossRef]

16. Sekiguchi, T.; Saika, A.; Nomura, K.; Watanabe, T.; Watanabe, T.; Fujimoto, Y.; Enoki, M.; Sato, T.; Kato, C.; Kanehiro, H. Biodegradation of aliphatic polyesters soaked in deep seawaters and isolation of poly(3-caprolactone)-degrading bacteria. Polym. Degrad. Stab. 2011, 96, 1397-1403. [CrossRef]

17. Tokiwa, Y.; Pranamuda, H. "Microbial Degradation of Aliphatic Polyesters" Biopolymers; Doi, Y., Steinbuchel, A., Eds.; Polyesters II Properties and Chemical Stnthesis; Wiley-VCH: Weinheim, Germany, 2002; Volume 3b, pp. 85-103.

18. Szuman, K.; Krucinska, I.; Bogun, M.; Draczynski, Z. PLA/PHB biodegradable blends for pneumothermic fablication of nonwoven. AUTEX Res. J. 2016, 16, 119-127. [CrossRef]

19. Sashiwa, H.; Kawasaki, N.; Nakaama, A.; Muraki, E.; Yamamoto, N.; Zhu, H.; Nagano, H.; Omura, Y.; Saimoto, H.; Shigemasa, Y.; et al. Chemical modification of chitosan 13. Synthesis of organosoluble, palladium adsorbable, and biodegradable chitosan derivertives toward the chemical plating on plastics. Biomacromolecules 2002, 3, 1120-1125. [CrossRef] [PubMed]

(C) 2018 by the authors. Licensee MDPI, Basel, Switzerland. This article is an open access article distributed under the terms and conditions of the Creative Commons Attribution (CC BY) license (http:/ / creativecommons.org/licenses/by/4.0/). 